\title{
Construction of Exact Solutions to Partial Differential Equations with CRE Method
}

\author{
Filiz Taşcan ${ }^{1 *}$, Arzu Akbulut ${ }^{2}$
}

\begin{abstract}
In this article, the consistent Riccati expansion (CRE) method is presented for constructing new exact solutions of (1+1) dimensional nonlinear dispersive modified Benjamin Bona Mahony (DMBBM) and mKdV-Burgers equations. The exact solutions obtained are composed of hyperbolic and exponential functions. The outcomes obtained confirm that the proposed method is an efficient technique for analytic treatment of a wide variety of nonlinear partial differential equations.
\end{abstract}

Keywords: Partial differential equations, Exact solution, The consistent Riccati expansion.

2010 AMS: Primary 35Q53, 35C07, Secondary 83C15

${ }^{1}$ Eskisehir Osmangazi University, Art-Science Faculty, Department of Mathematics-Computer, Eskisehir-TURKEY, ORCID:

0000-0003-2697-5271

${ }^{2}$ Eskisehir Osmangazi University, Art-Science Faculty, Department of Mathematics-Computer, Eskisehir-TURKEY, ORCID:

0000-0003-2448-2481

*Corresponding author: ftascan@ogu.edu.tr

Received: 21 November 2018, Accepted: 15 February 2019, Available online: 27 June 2019

\section{Introduction}

Nonlinear evolution equations (NLEEs) in mathematical physics play a vital role in different fields, such as fluid mechanics, plasma physics, optical fibers, solid state physics, chemical kinematic, chemical physics and geochemistry. Since obtaining exact solutions of NLEEs come into prominence, there become significant improvements in this domain[1]. Many effective and powerful methods have been established and improved, such as modified simple equation method [2], symmetry reduction method[3], trial equation method [4], the $\left(G^{\prime} / G\right)$-expansion method [5], sub equation method [6], $\exp (-\Phi(\xi))$ method[7], functional variable method[8], first integral method[9], modified exp-function method [10] and so on.

The aim of this paper is search new solutions of (1+1) dimensional nonlinear dispersive modified Benjamin Bona Mahony (DMBBM) equation and modified Korteweg-de Vries (mKdV)-Burgers equation with consistent Riccati expansion (CRE) method. In section 2, we give the definition of the method. In section 3, there found solutions of the given equations. In section 4 , conslusions are given.

\section{Consistent Riccati expansion (CRE) method}

Lets assume that we have a nonlinear differential equation, remark in the independent variables $x$ and $t$ and dependent variable $u$, given by

$$
F\left(u, u_{x}, u_{t}, u_{x x}, u_{t t}, \ldots\right)=0,
$$

where $F$ is a polynomial of $u(x, t)$ and its various partial derivatives including the highest order derivatives and nonlinear terms. 
According to the algoritm, we can seek for the solutions of Eq. (2.1) in the form

$$
u=\sum_{i=0}^{n} u_{i}(x, t) R^{i}(w)
$$

where $u_{i}(i=0, \ldots, n)$ are functions to be detected later and the positive integer $n$ can be detected by using homogeneous balance method. Here $R(w)$ is a solution of the Riccati equation

$$
R_{w}=a_{0}+a_{1} R+a_{2} R^{2}
$$

where $a_{0}, a_{1}, a_{2}$ are parameters to be determined and $w$ is an undetermined function of $x$ and $t$.

The positive integer $n$ can be detected by considering the homogeneous balance between the highest order derivative term with the highest order nonlinear term appearing in Eq. (2.1). Then by sett ing Eq. (2.2) along with Eq. (2.3) into Eq. (2.1) and equating the coefficients of all powers of $R(w)$ to zero yields a set of algebraic equations for unknowns $u_{i}, a_{0}, a_{1}$ and $a_{2}[11,12]$.

\section{Exercises}

In this part, we have dealed with two partial differential equations as an application of the CRE method.

\section{$3.1(1+1)$ dimensional nonlinear dispersive modified Benjamin Bona Mahony (DMBBM) equation}

Firstly, we look at the (1+1) dimensional nonlinear dispersive modified Benjamin Bona Mahony (DMBBM) equation [13]

$$
u_{t}+u_{x}-\alpha u^{2} u_{x}+u_{x x x}=0
$$

where where $\alpha$ is a nonzero constant. This equation was first derived to describe an approximation for surface long waves in nonlinear dispersive media. It can also characterize the hydro magnetic waves in cold plasma, acoustic waves in inharmonic crystals and acoustic gravity waves in compressible fluids [14].

Here, it is clear from the homogoneous balance principle that the balancing number is 1 . From here, we infer from that the exact solution of Eq. (3.1) is

$$
u(x, t)=u_{0}(x, t)+u_{1}(x, t) R(w(x, t))
$$

where $u_{0}(x, t)$ and $u_{1}(x, t)$ are functions to be determined later. Setting Eq. (3.2) and its derivatives with the condition Eq. (2.3) into Eq. (3.1) and gathering all terms with the same power of $R(w),(i=0,1, \ldots, 4)$, we obtain the following system

$$
\begin{aligned}
R^{4}(w): \quad & 6 u_{1} w_{x}^{3} a_{2}^{3}-\alpha u_{1}^{3} w_{x} a_{2}=0 \\
R^{3}(w): \quad & -2 \alpha u_{0} u_{1}^{2} w_{x} a_{2}+6\left(u_{1}\right)_{x} w_{x}^{2} a_{2}^{2}+12 u_{1} w_{x}^{3} a_{1} a_{2}^{2}+6 u_{1} w_{x} w_{x x} a_{2}^{2} \\
& -\alpha u_{1}^{2}\left(u_{1}\right)_{x}-\alpha u_{1}^{3} w_{x} a_{1}=0 \\
R^{2}(w): \quad & \\
& -\alpha u_{1}^{2}\left(u_{0}\right)_{x} w_{x}^{2} a_{1} a_{2}+8 u_{1} w_{x}^{3} a_{0} a_{2}^{2}-\alpha u_{0}^{2} u_{1} w_{x} a_{2}+9 u_{0} u_{1}^{2} w_{x} a_{1}+w_{x x} a_{1} a_{2} a_{2}-\alpha u_{1}^{3} w_{x} a_{0} \\
& +7 u_{1} w_{x}^{3} a_{1}^{2} a_{2}+3\left(u_{1}\right)_{x x} w_{x} a_{2}-2 \alpha u_{0} u_{1}\left(u_{1}\right)_{x} \\
& +3\left(u_{1}\right)_{x} w_{x x} a_{2}+u_{1} w_{x x x} a_{2}=0 \\
& \\
& \left(u_{1}\right)_{x x x}+u_{1} w_{t} a_{1}+3\left(u_{1}\right)_{x} w_{x x} a_{1}+3 u_{1} w_{x} w_{x x} a_{1}^{2} \\
& +\left(u_{1}\right)_{t}-\alpha u_{0}^{2} u_{1} w_{x} a_{1}+8 u_{1} w_{x}^{3} a_{1} a_{2} a_{0} \\
& -2 \alpha u_{0} u_{1}^{2} w_{x} a_{0}-2 \alpha u_{0} u_{1}\left(u_{0}\right)_{x}+u_{1} w_{x}^{3} a_{1}^{3}+\left(u_{1}\right)_{x} \\
& +6\left(u_{1}\right)_{x} w_{x}^{2} a_{2} a_{0}+6 u_{1} w_{x} w_{x x} a_{2} a_{0}+u_{1} w_{x x x} a_{1} \\
& +3\left(u_{1}\right)_{x x} w_{x} a_{1}+3\left(u_{1}\right)_{x} w_{x}^{2} a_{1}^{2}+u_{1} w_{x} a_{1}-\alpha u_{0}^{2}\left(u_{1}\right)_{x}=0 \\
& \\
& 3\left(u_{1}\right)_{x} w_{x}^{2} a_{1} a_{0}+u_{1} w_{x x x} a_{0}+3 u_{1} w_{x} w_{x x} a_{1} a_{0} \\
& +\left(u_{0}\right)_{x}+\left(u_{0}\right)_{t}+\left(u_{0}\right)_{x x x}+3\left(u_{1}\right)_{x x} w_{x} a_{0}+u_{1} w_{t} a_{0} \\
& +3\left(u_{1}\right)_{x} w_{x x} a_{0}-\alpha u_{0}^{2}\left(u_{0}\right)_{x}+u_{1} w_{x}^{3} a_{1}^{2} a_{0} \\
& +u_{1} w_{x} a_{0}+2 u_{1} w_{x}^{3} a_{2} a_{0}^{2}-\alpha u_{0}^{2} u_{1} w_{x} a_{0}=0 . \\
R^{0}(w) \quad 0 &
\end{aligned}
$$


From the Eq. (3.3), we get

$$
u_{1}(x, t)=\sqrt{6} \sqrt{\frac{1}{\alpha}} a_{2} w_{x} .
$$

If we substitute Eq. (3.8) in Eq. (3.4), we obtain

$$
u_{0}(x, t)=\frac{\sqrt{6} \sqrt{\frac{1}{\alpha}} w_{x x}}{2 w_{x}}+\sqrt{6} \sqrt{\frac{1}{\alpha}} a_{1} w_{x}-\frac{1}{2} w_{x} \alpha \sqrt{6} \sqrt{\left(\frac{1}{\alpha}\right)^{3}} a_{1} .
$$

When we substitute Eq. (3.8) and Eq. (3.9) in Eq. (3.5), we get following partial differential equation

$$
w_{t} w_{x}=-\frac{4 a_{2} w_{x}^{4} a_{0}-w_{x}^{4} a_{1}^{2}-3 w_{x x}^{2}+2 w_{x} w_{x x x}+2 w_{x}^{2}}{2} .
$$

If we use Eq. (3.10) in Eq. (3.6) and Eq. (3.7), these Eqs. are equal to zero.

If $w$ is a solution of Eq. (3.10), then

$$
u=\frac{\sqrt{6} \sqrt{\frac{1}{\alpha}} w_{x x}}{2 w_{x}}+\sqrt{6} \sqrt{\frac{1}{\alpha}} a_{1} w_{x}-\frac{1}{2} w_{x} \alpha \sqrt{6} \sqrt{\left(\frac{1}{\alpha}\right)^{3}} a_{1}+\sqrt{6} \sqrt{\frac{1}{\alpha}} a_{2} w_{x} R
$$

is a solution of the DMBBM equation with $R \equiv R(w)$ being a solution of the Riccati equation (2.3).

We suppose that $w(x, t)$ be of the form

$$
w(x, t)=a \cosh (k x+l t+\xi)+b \sinh (k x+l t+\xi)+r
$$

where $a, b, k, l$ and $r$ are constants to be determined later and $\xi$ is an arbitrary constant. Setting Eq. (3.12) into Eq. (3.10), we obtain the following equations

$$
\begin{gathered}
-\frac{k\left(16 a_{2} a_{0} a^{3} k^{3} b-4 a_{1}^{2} a k^{3} b^{3}-4 a_{1}^{2} a^{3} k^{3} b+16 a_{2} a_{0} a k^{3} b^{3}\right)}{2}=0, \\
-\frac{k\left(-16 a_{2} a_{0} a^{3} k^{3} b+4 a l b+4 a_{1}^{2} a^{3} k^{3} b-2 a k^{3} b+4 a k b\right)}{2}=0, \\
-\frac{k\left(-a_{1}^{2} a^{4} k^{3}+24 a_{2} a_{0} a^{2} k^{3} b^{2}-a_{1}^{2} b^{4} k^{3}\right)}{2} \\
-\frac{k\left(-6 a_{1}^{2} a^{2} k^{3} b^{2}+4 a_{2} a_{0} b^{4} k^{3}+4 a_{2} a_{0} a^{4} k^{3}\right)}{2}=0, \\
-\frac{k\left(2 a_{1}^{2} a^{4} k^{3}+6 a_{1}^{2} a^{2} k^{3} b^{2}-8 a_{2} a_{0} a^{4} k^{3}+2 a^{2} k+2 b^{2} k\right)}{2} \\
-\frac{k\left(-b^{2} k^{3}-a^{2} k^{3}+2 a^{2} l-24 a_{2} a_{0} a^{2} k^{3} b^{2}+2 b^{2} l\right)}{2}=0, \\
-\frac{k\left(-a_{1}^{2} a^{4} k^{3}-2 a^{2} k-2 a^{2} l-2 a^{2} k^{3}+4 a_{2} a_{0} a^{4} k^{3}+3 b^{2} k^{3}\right)}{2}=0
\end{gathered}
$$

Solving above system, we get the following two solutions.

State 1:

$$
\begin{aligned}
& a=b, a_{0}=\frac{a_{1}^{2}}{4 a_{2}}, a_{1}=a_{1}, a_{2}=a_{2}, b=b, \\
& k=k, \xi=\xi, l=\frac{k\left(k^{2}-2\right)}{2}, r=r .
\end{aligned}
$$

State 2:

$$
\begin{aligned}
& a=-b, a_{0}=\frac{a_{1}^{2}}{4 a_{2}}, a_{1}=a_{1}, a_{2}=a_{2}, b=b, \\
& k=k, \xi=\xi, l=\frac{k\left(k^{2}-2\right)}{2}, r=r .
\end{aligned}
$$


Combining Eq. (3.11), Eq. (3.12) with Eq. (3.13) and Eq. (3.14), two families of exact explicit solutions to the DMBBM equation are obtained

$$
\begin{aligned}
u(x, t)= & \frac{1}{2} \sqrt{6} \sqrt{\frac{1}{\alpha}} k\left(a_{1} b \cosh (\beta)+1+a_{1} b \sinh (\beta)\right) \\
& +\sqrt{6} \sqrt{\frac{1}{\alpha}} a_{2}(b k \sinh (\beta)+b k \cosh (\beta)) \\
& \times R(b \cosh (\beta)+b \sinh (\beta)+r)
\end{aligned}
$$

and

$$
\begin{aligned}
u(x, t)= & \frac{1}{2} \sqrt{6} \sqrt{\frac{1}{\alpha}} k\left(a_{1} b \cosh (\beta)-1-a_{1} b \sinh (\beta)\right) \\
& +\sqrt{6} \sqrt{\frac{1}{\alpha}} a_{2}(-b k \sinh (\beta)+b k \cosh (\beta)) \\
& \times R(-b \cosh (\beta)+b \sinh (\beta)+r) .
\end{aligned}
$$

where $\beta=k x-k t+\frac{k^{3} t}{2}+\xi$.

We suppose that $w(x, t)$ be of the form

$$
w(x, t)=A \exp \left(k_{1} x+l_{1} t+\xi_{1}\right)+B \exp \left(k_{2} x+l_{2} t+\xi_{2}\right)+C
$$

where $A, B, C, k_{i}$ and $l_{i}$ are constants to be determined later and $\xi_{i}$ are an arbitrary constant. Setting Eq. (3.15) into Eq. (3.10), we get the following system

$$
\begin{aligned}
& \frac{a_{1}^{2} B^{4} k_{2}^{4}}{2}-2 a_{2} a_{0} B^{4} k_{2}^{4}=0, \\
&-8 a_{2} a_{0} A k_{1} B^{3} k_{2}^{3}+2 a_{1}^{2} A k_{1} B^{3} k_{2}^{3}=0,=0,-12 a_{2} a_{0} A^{2} k_{1}^{2} B^{2} k_{2}^{2}+3 a_{1}^{2} A^{2} k_{1}^{2} B^{2} k_{2}^{2}=0, \\
&-B^{2} k_{2}^{2}+\frac{1}{2} B^{2} k_{2}^{4}-B^{2} l_{2} k_{2}=0, \\
&-8 a_{2} a_{0} A^{3} k_{1}^{3} B k_{2}+2 a_{1}^{2} A^{3} k_{1}^{3} B k_{2}=0, \\
&-A l_{1} B k_{2}-A k_{1}^{3} B k_{2}-B l_{2} A k_{1}-B k_{2}^{3} A k_{1}+3 A k_{1}^{2} B k_{2}^{2}-2 A k_{1} B k_{2}=0, \\
& \frac{a_{1}^{2} A^{4} k_{1}^{4}}{2}-2 a_{2} a_{0} A^{4} k_{1}^{4}=0, \\
& \frac{A^{2} k_{1}^{4}}{2}-A^{2} k_{1}^{2}-A^{2} l_{1} k_{1}=0 .
\end{aligned}
$$

Solving above system, one gets the following set of solution.

$$
\begin{aligned}
& A=A, B=B, C=C, a_{0}=\frac{a_{1}^{2}}{4 a_{2}}, a_{1}=a_{1}, a_{2}=a_{2}, k_{1}=k_{2}, \\
& k_{2}=k_{2}, l_{1}=-k_{2}+\frac{k_{2}^{3}}{2}, l_{2}=-k_{2}+\frac{k_{2}^{3}}{2}, \xi_{1}=\xi_{1}, \xi_{2}=\xi_{2}
\end{aligned}
$$


Combining Eq. (3.11), Eq. (3.15) with Eq. (3.16), exact explicit solution is obtained

$$
\begin{aligned}
u(x, t)= & \frac{k_{2} \sqrt{6}}{2} \sqrt{\frac{1}{\alpha}}\left(A a_{1} \exp \left(\beta+\xi_{1}\right)+B a_{1} \exp \left(\beta+\xi_{2}\right)+1\right) \\
& +a_{2} \sqrt{6} \sqrt{\frac{1}{\alpha}}\left(A k_{2} \exp \left(\beta+\xi_{1}\right)+B k_{2} \exp \left(\beta+\xi_{2}\right)\right) \\
& \times R\left(A \exp \left(\beta+\xi_{1}\right)+B \exp \left(\beta+\xi_{2}\right)+C\right)
\end{aligned}
$$

where $\beta=k_{2} x+\left(-k_{2}+\frac{k_{2}^{3}}{2}\right) t$.

\subsection{Modified Korteweg-de Vries (mKdV)-Burgers equation}

$\mathrm{mKdV}$-Burgers equation is given by [15]

$$
u_{t}+q u^{2} u_{x}+r u_{x x}-s u_{x x x}=0
$$

where $q, r$ and $s$ are arbitrary constants. According to the homogeneous balance method, we get the balancing number as $n=1$. From here, we infered that the exact solution of Eq. (3.17) is

$$
u(x, t)=u_{0}(x, t)+u_{1}(x, t) R(w(x, t))
$$

where $u_{0}(x, t)$ and $u_{1}(x, t)$ are functions to be detected later. Setting Eq. (3.18) and its derivatives with the condition Eq. (2.3) into Eq. (3.17) and picking all terms with the same power of $R(w),(i=0,1, \ldots, 4)$, we have the following system

$$
\begin{aligned}
R^{4}(w): & q u_{1}^{3} w_{x} a_{2}-6 s u_{1} w_{x}^{3} a_{2}^{3}=0 \\
R^{3}(w): \quad & 2 q u_{0} u_{1}^{2} w_{x} a_{2}-6 s\left(u_{1}\right)_{x} w_{x}^{2} a_{2}^{2}+2 r u_{1} w_{x}^{2} a_{2}^{2}-12 s u_{1} w_{x}^{3} a_{1} a_{2}^{2} \\
& +q u_{1}^{2}\left(u_{1}\right)_{x}+q u_{1}^{3} w_{x} a_{1}-6 s u_{1} w_{x} w_{x x} a_{2}^{2}=0 \\
R^{2}(w): \quad & q u_{1}^{2}\left(u_{0}\right)_{x}+2 r\left(u_{1}\right)_{x} w_{x} a_{2}-s u_{1} w_{x x x} a_{2} \\
& +u_{1} w_{t} a_{2}-3 s\left(u_{1}\right)_{x} w_{x x} a_{2}-8 s u_{1} w_{x}^{3} a_{0} a_{2}^{2} \\
& +3 r u_{1} w_{x}^{2} a_{1} a_{2}+2 q u_{0} u_{1}^{2} w_{x} a_{1}-3 s\left(u_{1}\right)_{x x} w_{x} a_{2} \\
& +2 q u_{0} u_{1}\left(u_{1}\right)_{x}-9 s\left(u_{1}\right)_{x} w_{x}^{2} a_{1} a_{2}-9 s u_{1} w_{x} w_{x x} a_{1} a_{2} \\
& +q u_{1}^{3} w_{x} a_{0}+q u_{0}^{2} u_{1} w_{x} a_{2}-7 s u_{1} w_{x}^{3} a_{1}^{2} a_{2}+r u_{1} w_{x x} a_{2}=0 \\
& \\
R^{1}(w): \quad & q u_{0}^{2}\left(u_{1}\right)_{x}-6 s u_{1} w_{x} w_{x x} a_{2} a_{0}+2 q u_{0} u_{1}^{2} w_{x} a_{0}+r\left(u_{1}\right)_{x x} \\
& +r u_{1} w_{x x} a_{1}-s u_{1} w_{x x x} a_{1}+\left(u_{1}\right)_{t}-3 s\left(u_{1}\right)_{x x} w_{x} a_{1} \\
& -3 s\left(u_{1}\right)_{x} w_{x}^{2} a_{1}^{2}-s u_{1} w_{x}^{3} a_{1}^{3}+r u_{1} w_{x}^{2} a_{1}^{2}-3 s\left(u_{1}\right)_{x} w_{x x} a_{1} \\
& +q u_{0}^{2} u_{1} w_{x} a_{1}-s\left(u_{1}\right)_{x x x}+u_{1} w_{t} a_{1}-3 s u_{1} w_{x} w_{x x} a_{1}^{2} \\
& -8 s u_{1} w_{x}^{3} a_{1} a_{2} a_{0}+2 r\left(u_{1}\right)_{x} w_{x} a_{1}+2 r u_{1} w_{x}^{2} a_{2} a_{0} \\
& +2 q u_{0} u_{1}\left(u_{0}\right)_{x}-6 s\left(u_{1}\right)_{x} w_{x}^{2} a_{2} a_{0}=0 \\
& \\
R^{0}(w):= & u_{1} w_{t} a_{0}-3 s\left(u_{1}\right)_{x} w_{x}^{2} a_{1} a_{0}+2 r\left(u_{1}\right)_{x} w_{x} a_{0}+r u_{1} w_{x}^{2} a_{1} a_{0} \\
& +r u_{1} w_{x x} a_{0}-3 s\left(u_{1}\right)_{x x} w_{x} a_{0}+q u_{0}^{2} u_{1} w_{x} a_{0}-s u_{1} w_{x x x} a_{0} \\
& +r\left(u_{0}\right)_{x x}-2 s u_{1} w_{x}^{3} a_{2} a_{0}^{2}-3 s\left(u_{1}\right)_{x} w_{x x} a_{0}+q u_{0}^{2}\left(u_{0}\right)_{x} \\
& -3 s u_{1} w_{x} w_{x x} a_{1} a_{0}+\left(u_{0}\right)_{t}-s\left(u_{0}\right)_{x x x}-s u_{1} w_{x}^{3} a_{1}^{2} a_{0}=0 .
\end{aligned}
$$

From the Eq. (3.19), we get

$$
u_{1}(x, t)=\frac{\sqrt{6} \sqrt{s} a_{2} w_{x}}{\sqrt{q}} .
$$


If we substitute Eq. (3.24) in Eq. (3.20), we obtain

$$
u_{0}(x, t)=\frac{\sqrt{6}\left(3 s w_{x x}-r w_{x}+3 s w_{x}^{2} a_{1}\right)}{6 \sqrt{s} \sqrt{q} w_{x}}
$$

When we substitute Eq. (3.24) and Eq. (3.25) in Eq. (3.21), we get following partial differential equation

$$
w_{t} w_{x}=-3 s w_{x x}^{2}+2 s a_{2} a_{0} w_{x}^{4}-\frac{s w_{x}^{4} a_{1}^{2}}{2}+s w_{x x x} w_{x}-\frac{w_{x}^{2} r^{2}}{6 s}
$$

If we use Eq. (3.26) in Eq. (3.22), this Eq. is equal to zero. If we use Eq. (3.26) in Eq. (3.23), we obtain

$$
\frac{r \sqrt{6 s}\left(4 w_{x x} a_{2} w_{x}^{4} a_{0}+3 w_{x x}^{3}+w_{x x x x} w_{x}^{2}-4 w_{x x} w_{x x x} w_{x}-w_{x}^{4} a_{1}^{2} w_{x x}\right)}{2 \sqrt{q} w_{x}^{3}}=0
$$

If $w$ is a solution of Eqs. (3.26) and (3.27), then

$$
u=\frac{\sqrt{6}\left(3 s w_{x x}-r w_{x}+3 s w_{x}^{2} a_{1}\right)}{6 \sqrt{s} \sqrt{q} w_{x}}+\frac{\sqrt{6} \sqrt{s} a_{2} w_{x}}{\sqrt{q}} R
$$

is a solution of the Eq. (3.17) with $R \equiv R(w)$ being a solution of the Riccati equation (2.3).

We suppose that $w(x, t)$ be of the form

$$
w(x, t)=a \cosh (k x+l t+\xi)+b \sinh (k x+l t+\xi)+r
$$

where $a, b, k, l$ and $r$ are constants to be determined later and $\xi$ is an arbitrary constant. Setting Eq. (3.29) into Eqs. (3.26) and 
(3.27), we obtain the following equations

$$
\begin{aligned}
& s a^{2} k^{4}-a^{2} k l-\frac{3 s b^{2} k^{4}}{2}-\frac{r^{2} a^{2} k^{2}}{6 s}=0,2 s a_{2} a_{0} a^{4} k^{4}-\frac{s a_{1}^{2} a^{4} k^{4}}{2}=0, \\
& -3 s a_{1}^{2} a^{2} k^{4} b^{2}+12 s a_{2} a_{0} a^{2} k^{4} b^{2}=0,8 s a_{2} a_{0} a^{3} k^{4} b-2 s a_{1}^{2} a^{3} k^{4} b=0, \\
& -\frac{s a_{1}^{2} b^{4} k^{4}}{2}+2 s a_{2} a_{0} b^{4} k^{4}=0,-s a k^{4} b-2 a k b l-\frac{r^{2} a k^{2} b}{3 s}=0, \\
& \frac{2 \sqrt{6} r \sqrt{s} b k^{6} a_{2} a^{4} a_{0}}{\sqrt{q}}-\frac{\sqrt{6} r \sqrt{s} a^{4} k^{6} b a_{1}^{2}}{2 \sqrt{q}}=0,-\frac{r^{2} b^{2} k^{2}}{6 s}-b^{2} k l-\frac{3 s a^{2} k^{4}}{2}+s b^{2} k^{4}=0, \\
& -\frac{\sqrt{6} r \sqrt{s} a^{5} k^{6} a_{1}^{2}}{2 \sqrt{q}}-\frac{2 \sqrt{6} r \sqrt{s} a^{3} k^{6} b^{2} a_{1}^{2}}{\sqrt{q}}+2 a_{0}\left(\frac{4 \sqrt{6} r \sqrt{s} a^{3} k^{6} b^{2} a_{2}}{\sqrt{q}}+\frac{\sqrt{6} r \sqrt{s} a^{5} k^{6} a_{2}}{\sqrt{q}}\right)=0, \\
& -\frac{2 \sqrt{6} r \sqrt{s} a^{4} k^{6} b a_{1}^{2}}{\sqrt{q}}-\frac{3 \sqrt{6} r \sqrt{s} a^{2} k^{6} b^{3} a_{1}^{2}}{\sqrt{q}}+2 a_{0}\left(\frac{6 \sqrt{6} r \sqrt{s} a^{2} k^{6} b^{3} a_{2}}{\sqrt{q}}+\frac{4 \sqrt{6} r \sqrt{s} a^{4} k^{6} a_{2} b}{\sqrt{q}}\right)=0, \\
& -2 s a_{1}^{2} a k^{4} b^{3}+8 s a_{2} a_{0} a k^{4} b^{3}=0,-\frac{3 \sqrt{6} r \sqrt{s} b k^{6} a^{2}}{2 \sqrt{q}}+\frac{3 \sqrt{6} r \sqrt{s} b^{3} k^{6}}{2 \sqrt{q}}=0, \\
& -\frac{3 \sqrt{6} r \sqrt{s} a^{3} k^{6} b^{2} a_{1}^{2}}{\sqrt{q}}-\frac{2 \sqrt{6} r \sqrt{s} a b^{4} k^{6} a_{1}^{2}}{\sqrt{q}}+2 a_{0}\left(\frac{4 \sqrt{6} r \sqrt{s} a k^{6} b^{4} a_{2}}{\sqrt{q}}+\frac{6 \sqrt{6} r \sqrt{s} a^{3} k^{6} a_{2} b^{2}}{\sqrt{q}}\right)=0, \\
& \frac{7 \sqrt{6} r \sqrt{s} a k^{6} b^{2}}{2 \sqrt{q}}+\frac{\sqrt{6} r \sqrt{s} a^{3} k^{6}}{2 \sqrt{q}}-2 a k\left(\frac{\sqrt{6} r \sqrt{s} k^{5} b^{2}}{\sqrt{q}}+\frac{\sqrt{6} r \sqrt{s} a^{2} k^{5}}{\sqrt{q}}\right)=0, \\
& -\frac{2 \sqrt{6} r \sqrt{s} a^{2} k^{6} b^{3} a_{1}^{2}}{\sqrt{q}}-\frac{\sqrt{6} r \sqrt{s} a b^{5} k^{6} a_{1}^{2}}{2 \sqrt{q}}+2 a_{0}\left(\frac{\sqrt{6} r \sqrt{s} b^{5} k^{6} a_{2}}{\sqrt{q}}+\frac{4 \sqrt{6} r \sqrt{s} a^{2} k^{6} a_{2} b^{3}}{\sqrt{q}}\right)=0, \\
& \frac{7 \sqrt{6} r \sqrt{s} b k^{6} a^{2}}{2 \sqrt{q}}+\frac{\sqrt{6} r \sqrt{s} b^{3} k^{6}}{2 \sqrt{q}}-2 b k\left(\frac{\sqrt{6} r \sqrt{s} k^{5} b^{2}}{\sqrt{q}}+\frac{\sqrt{6} r \sqrt{s} a^{2} k^{5}}{\sqrt{q}}\right)=0, \\
& \frac{3 \sqrt{6} r \sqrt{s} a^{3} k^{6}}{2 \sqrt{q}}-\frac{3 \sqrt{6} r \sqrt{s} a k^{6} b^{2}}{2 \sqrt{q}}=0, \frac{2 \sqrt{6} r \sqrt{s} a k^{6} b^{4} a_{2} a_{0}}{\sqrt{q}}-\frac{\sqrt{6} r \sqrt{s} a b^{4} k^{6} a_{1}^{2}}{2 \sqrt{q}}=0,
\end{aligned}
$$

Solving above system, we get the following two solutions.

\section{State 1:}

$$
\begin{aligned}
& a=b, a_{0}=\frac{a_{1}^{2}}{4 a_{2}}, a_{1}=a_{1}, a_{2}=a_{2}, b=b, \\
& k=k, \xi=\xi, l=-\frac{k\left(3 s^{2} k^{2}+r^{2}\right)}{6 s}, r=r .
\end{aligned}
$$

\section{State 2:}

$$
\begin{aligned}
& a=-b, a_{0}=\frac{a_{1}^{2}}{4 a_{2}}, a_{1}=a_{1}, a_{2}=a_{2}, b=b, \\
& k=k, \xi=\xi, l=-\frac{k\left(3 s^{2} k^{2}+r^{2}\right)}{6 s}, r=r .
\end{aligned}
$$

Combining Eq. (3.28), Eq. (3.29) with Eq. (3.30) and Eq. (3.31), two families of exact explicit solutions to the mKdV-Burgers 
equation are obtained

$$
\begin{aligned}
u(x, t)= & \frac{\sqrt{6} b^{3} k^{3}(\cosh (\beta)-\sinh (\beta))^{3}\left(3 s k-r+3 s b k a_{1} \cosh (\beta)-3 s b k a_{1} \sinh (\beta)\right)}{6 \sqrt{s} \sqrt{q}(-b k \sinh (\alpha)+b k \cosh (\alpha))^{3}} \\
& +\frac{\sqrt{6 s}}{\sqrt{q}}(-b k \sinh (\alpha)+b k \cosh (\alpha)) a_{2} R(b \cosh (\alpha)-b \sinh (\alpha)+r)
\end{aligned}
$$

and

$$
\begin{aligned}
u(x, t)= & \frac{\sqrt{6} b^{3} k^{3}(\cosh (\beta)-\sinh (\beta))^{3}\left(-3 s k-r+3 s b k a_{1} \cosh (\beta)+3 s b k a_{1} \sinh (\beta)\right)}{6 \sqrt{s} \sqrt{q}(b k \sinh (\alpha)+b k \cosh (\alpha))^{3}} \\
+ & \frac{\sqrt{6 s}}{\sqrt{q}}(b k \sinh (\alpha)+b k \cosh (\alpha)) a_{2} R(-b \cosh (\alpha)-b \sinh (\alpha)+r)
\end{aligned}
$$

where $\alpha=-k x+\frac{k\left(3 s^{2} k^{2}+r^{2}\right) t}{6 s}-\xi, \beta=\frac{-6 k x s+3 k^{3} t s^{2}+k t r^{2}-6 s \xi}{6 s}$.

We suppose that $w(x, t)$ be of the form

$$
w(x, t)=A+B \exp \left(k_{1} x+l_{1} t+\xi_{1}\right)
$$

where $A, B, k_{1}$ and $l_{1}$ are constants to be determined later and $\xi_{1}$ are an arbitrary constant. Setting Eq. (3.32) into Eqs. (3.26) and (3.27), we get the following system

$$
\begin{array}{r}
\frac{2 \sqrt{6} r \sqrt{s} B^{2} k_{1}^{3} a_{2} a_{0}}{\sqrt{q}-\frac{\sqrt{6} r \sqrt{s} B^{2} k_{1}^{3} a_{1}^{2}}{2 \sqrt{q}}}=0, \\
2 s B^{3} k_{1}^{3} a_{2} a_{0}-\frac{s B^{3} k_{1}^{3} a_{1}^{2}}{2}=0, \\
-\frac{s B k_{1}^{3}}{2}-\frac{B k_{1} r^{2}}{6 s}-B l_{1}=0,
\end{array}
$$

Solving above system, one gets the following set of solution

$$
\begin{aligned}
& A=A, B=B, a_{0}=\frac{a_{1}^{2}}{4 a_{2}}, a_{1}=a_{1}, a_{2}=a_{2}, \\
& k_{1}=k_{1}, l_{1}=-\frac{k_{1}\left(3 s^{2} k_{1}^{2}+r^{2}\right)}{6 s}, \xi_{1}=\xi_{1} .
\end{aligned}
$$

Combining Eq. (3.28), Eq. (3.29) with Eq. (3.33), exact explicit solution is obtained

$$
\begin{aligned}
u(x, t)= & \frac{\sqrt{6} \exp (\alpha)\left(3 k_{1} s-r+3 s B k_{1} a_{1} \exp \left(\frac{\alpha}{3}\right)\right)}{6 \sqrt{s q}(\exp (\beta))^{3}} \\
& +\frac{\sqrt{6} \sqrt{s} B k_{1} a_{2} \exp (\beta) R(A+B \exp (\beta))}{\sqrt{q}}
\end{aligned}
$$

where $\alpha=-\frac{-6 k_{1} x s+3 k_{1}^{3} t s^{2}+k_{1} t r^{2}-6 \xi_{1} s}{2 s}, \beta=k_{1} x-\frac{k_{1}\left(3 s^{2} k_{1}^{2}+r^{2}\right) t}{6 s}+\xi_{1}$

\section{Conclusions}

In this paper, by introducing CRE method we apply to DMBBM and $\mathrm{mKdV}$-Burgers equations. We had exact explicit solutions of given equations with the help of Riccati equation. The obtained exact solutions are consist of hyperbolic and exponential functions. We checked all solutions of given equations by the Maple.

It is also shown that the CRE method can be performed to other kinds of integrable systems and can be obtained other kind of solutions. 


\section{References}

[1] M. Kaplan, A. Akbulut, A. Bekir, Exact travelling wave solutions of the nonlinear evolution equations by auxiliary equation method, Z. Naturforsch A, 70 (2015), 969-974.

[2] A. Bekir, A. Akbulut, M. Kaplan, Exact solutions of nonlinear evolution equations by using modified simple equation method, Int. J. Nonlinear Sci., 19 (2015), 159-164.

[3] F. Taşcan, A. Yakut, Conservation laws and exact solutions with symmetry reduction of nonlinear reaction diffusion equations, Int. J. Nonlinear Sci. Numer. Simul., 16 (2015), 191-196.

[4] M. Ekici, M. Mirzazadeh, Q. Zhou, S. P. Moshokoa, A. Biswas, M. Belic, Solitons in optical metamaterials with fractional temporal evolution, Optik, 127 (2016), 10879-10897.

[5] M. Mirzazadeh, M. Eslami, D. Milovic, A. Biswas, Topological solitons of resonant nonlinear Schödinger'sequation with dual-power law nonlinearity by $\left(G^{\prime} / G\right)$-expansion technique, Optik, 125 (19), (2014) 5480-5489.

[6] Q. Feng, F. Meng, Explicit solutions for space-time fractional partial differential equations in mathematical physics by a new generalized fractional Jacobi elliptic equation-based sub-equation method, Optik, 127 (2016), $7450-7458$.

[7] A. Akbulut, M. Kaplan, F. Taşcan, The investigation of exact solutions of nonlinear partial differential equations by using $\exp (-\Phi(\xi))$ method, Optik, 132 (2017), 382-387.

[8] A. Biswas, M. Mirzazadeh, M. Eslami, D. Milovic, M. Belic, Solitons in optical metamaterials by functional variable method and first integral approach, Frequenz, 68 (11-12) (2014), 525-530.

[9] B. Lu, The first integral method for some time fractional differential equations, J. Math. Anal. Appl., 395 (2012), 684-693.

[10] H.M. Başkonuş, H. Bulut, Analytical studies on the (1+1)-dimensional nonlinear Dispersive Modified Benjamin-BonaMahony equation defined by seismic sea waves, Waves Random Complex Media, doi:10.1080/17455030.1062577, 2015.

[11] J. Xiang-Li, L. Sen-Yue, CRE method for solving $m K d V$ equation and new interactions between solitons and cnoidal periodic waves, Commun. Theor. Phys., 63 (2015), 7-9.

[12] M. Chen, H. Hu, H. Zhu, Consistent Riccati expansion and exact solutions of the Kuramoto-Sivashinsky equation, Appl. Math. Lett., 49 (2015), 147-151.

[13] K. Khan, M.A. Akbar, S.M. Raynaul Islam, Exact solutions for $(1+1)$-dimensional nonlinear dispersive modified Benjamin-Bona-Mahony equation and coupled Klein-Gordon equations, SpringerPlus, 3 (2014), 8 pages.

[14] E.M.E. Zayed, S. Al-Joudi, Applications of an extended (G'/G)-expansion method to find exact solutions of nonlinear PDEs in mathematical physics, Math. Probl. Eng., Article ID 768573, doi:10.1155/2010/768573, (2010), 19 pages.

[15] M. Wang, Exact solutions for a compound KdV-Burgers equation, Phys. Lett. A., 213 (1996), 279-287. 